\title{
Preparation and Stability Evaluation of Pectin Coated Galanga Essential Oil Liposomes
}

\author{
Yupo Cao ${ }^{a}$, Wei Zhou ${ }^{\mathrm{a}, *}$, Jihua $\mathrm{Li}^{\mathrm{a}}$, Xiaobing Huang ${ }^{\mathrm{a}}$, Yuan Yuan ${ }^{\mathrm{a}}$, Lijing Lin ${ }^{\mathrm{a}}$ \\ ${ }^{a}$ Agricultural Products Processing Research Institute, Chinese Academy of Tropical Agricultural \\ Sciences, Zhanjiang 524000, China
}

Key words: Galanga essential oil; Liposomes; Pectin-coated; Stability

\begin{abstract}
Galanga essential oil liposomes were prepared by ethanol injection method, and uesd pectin to coat galanga essential oil liposomes. The average diameter, PDI, zeta potential, entrapment efficiency and storage stability of pectin-coated galanga essential oil liposomes were investigated. Results showed that pectin-coated galanga essential oil liposomes (concentration of pectin $(0.3 \%$, w/v)) had similar entrapment efficiency $(32.81 \pm 3.72 \% \sim 35.58 \pm 3.53 \%)$, and the average diameter of liposomes generally increased from $65.72 \pm 5.81 \mathrm{~nm}$ to $125.62 \pm 13.35 \mathrm{~nm}$, the zeta potential values of liposomes decreased from $-3.31 \pm 1.12 \mathrm{mV}$ to $-35.53 \pm 2.45 \mathrm{mV}$. Then stored at $4{ }^{\circ} \mathrm{C}$ and $25^{\circ} \mathrm{C}$ for 10 weeks, the pectin-coated galanga essential oil liposomes showed better storage stability, with the little change of average diameter and leakage of galanga essential oil.
\end{abstract}

\section{Introduction}

Galanga is a member of the Zingiberaceae family which is a pungent and aromatic dry rhizome. It was found that galangal had various pharmacological activities including anticancer, anti-oxidant, antihypertensive, anti-inflammatory and antibacterial [1]. This was mainly related to its chemical composition galangal essential oil. Galangal essential oil is one of the secondary metabolites which exists in galangal rhizome and is an aromatic and volatile oily liquid [2]. Pharmacological study showed that the galangal essential oil has antimicrobial activity and antioxidant effect, but its maintenance phase of antimicrobial activity is pretty short due to the unstability and effumability.

Liposomes, which were spontaneous formation of the phospholipids dispersion in water double vesicles, have been widely used in pharmaceutical, food, and cosmetics field [3]. This double-layer structure of liposomes given unique amphiphilic properties, phosphatide double molecular hydrophobic area can package material and the water phase kernel can package hydrophilic drugs [4]. Liposomes have many excellent properties, such as biocompatibility, targeting and slow-releasing potential [5]. However, it still has many problems such as drug leakage, bigger particle size, lipid oxidation after long-term storage when pure essential oil was entrapped to liposomes.

This study was aimed to solve the problem of unstable galangal essential oil and liposomes. Pectin was used to coat the galangal essential oil liposomes, then its basic properties such as particle size, zeta potential, entrapment efficiency were measured, and its storage stability was also studied. This research can improve the stability of galangal essential oil and can provide a theoretical guidance for based on natural preservatives to improve the stability of the liposomes carrier.

\section{Materials and methods}

\subsection{Materials}

Galangal essential oil was provided by the Agricultural Products Processing Institute of the Chinese Academy of Tropical Agricultural Sciences (Zhanjiang, China). Pectin was extracted from creeping fig (Ficus pumila Linn.) seeds. Phospholipid S75 was purchased from Lipoid GmbH (Germany). Cholesterol was obtained from Sigma-Aldrich Chemical Co. (USA). Ethanol, Tween 80, Vitamin E and other materials were all of analytical grade. Phosphate buffer (pH7.4).

\footnotetext{
${ }^{*}$ Corresponding author. Wei Zhou, Agricultural Products Processing Research Institute, Chinese Academy of Tropical
} Agricultural Sciences, Zhanjiang 524001, China. Tel: +86 759 2221090. E-mail address: weizhou111@ foxmail.com. 


\subsection{Methods}

\subsubsection{Preparation of GEO nanoliposomes}

The mass ratio of galangal essential oil, VE, tween 80, phospholipid and cholesterol was 60: 8: 15: 1.2: 10. They were dissolved in sufficient dehydrated alcohol with a magnetic stirrer (IKARCT, Guangzhou, China). The obtained ethanol solution was injected slowly into phosphate-buffered saline $(\mathrm{pH} 7.4,0.05 \mathrm{M})$ using a syringe pump and stirred vigorously for 30 minutes. The obtained suspension was transferred to an appropriate round bottom flask and submitted to a rotary evaporator (RE-2000, Yarong Biochemistry Installation Factory, Shanghai, China) at a proper temperature to evaporate the dehydrated alcohol. The final concentration of galangal essential oil is $1 \mathrm{mg} / \mathrm{mL}$. GEO nanoliposomes was obtained by the super-high pressure viscolizer (micro-jet, Langfang general machine Ltd, Langfang, China) at a pressure of $100 \mathrm{MPa}$.

\subsubsection{Preparation of pectin-coated GEO liposomes}

Firstly, the pectin was dissolved in phosphate buffer $(50 \mathrm{mM}, \mathrm{pH} 6.8)$ under stirring at room temperature overnight. The pectin-coated GEO nanoliposomes (PNL) were prepared by adding GEO nanoliposomes into pectin solutions at the ratio of $1: 1(\mathrm{v} / \mathrm{v})$ under continuous magnetic stirring at room temperature for 1 hour. The final concentration of pectin was adjusted to $0.1 \%, 0.2 \%, 0.3 \%, 0.4 \%$ and $0.5 \%(\mathrm{w} / \mathrm{v})$. The control group was prepared by adding GEO nanoliposomes to phosphate buffer at the same condition, named NL.

\subsubsection{Characterization of GEO nanoliposomes (NL) and pectin-coated GEO liposomes (PNL)}

\subsubsection{Determination of Entrapment Efficiency}

The determination of entrapment efficiency (EE, \%) of GEO in the NL and PNL was according to the method of organic solvent extraction. Briefly, the sample was infunded in a test tube, and add $3 \mathrm{~mL}$ petroleum ether, after mixing, let stand for $30 \mathrm{~min}$. After 3 times of extraction, the upper organic phase was transferred to the $25 \mathrm{~mL}$ volumetric flask, and use the anhydrous ethanol constant volume. Then the amount of free GEO (Ws) was assayed by UV-Visible spectrophotometer (T6, Beijing Purkinje General Instrument Co., China), and the amount of total GEO was Wo, so the entrapment efficiency of GEO (EE) was calculated from equation (1). Experiments were performed in triplicate. $\mathrm{EE}=\frac{\mathrm{Wo}-\mathrm{Ws}}{\mathrm{Wo}} \times 100 \%$

\subsubsection{Determination of particle size and polydispersity index}

The appropriate NL and PNL samples were diluted 10-fold with distilled water and stirred using a vortex mixing oscillation apparatus (Guangzhou, China) before determination. Size and PDI of Samples were measured by the laser particle size analyser (Nicomp 380/ZLS, Pss Corporation, USA) at $25^{\circ} \mathrm{C}$. Samples were scattered at $635 \mathrm{~nm}$ (angle of 90 degrees). Each sample was measured in triplicate.

\subsubsection{Determination of zeta potential}

By setting refraction (1.333), viscosity $(0.933)$ and laser wavelength $(635 \mathrm{~nm})$, zeta potential values were measured using a laser particle size analyser at $25^{\circ} \mathrm{C}$. All determinations were carried out in triplicate.

\subsubsection{Storage stability study}

The NL and PNL were stored at $4{ }^{\circ} \mathrm{C}$ and $25^{\circ} \mathrm{C}$ for 10 weeks in a sealed and dark condition, respectively. The average diameter, zeta potential, and leakage ratio of GEO were determined every week. The leakage ratio of GEO was calculated according to the following equation (2). Each experiment was analyzed in triplicate.

leakage ratio $=\left(1-\frac{E_{\text {during the storage time }}}{E_{\text {before storage }}}\right) \times 100 \%$

\subsection{Statistical Analysis}

All experiments were done in triplicate unless otherwise specified and the values are expressed as means \pm S.D. Data were subjected to statistical analysis by Student's t-test. Differences were considered significant at $\mathrm{p}<0.05$. 


\section{Results and discussion}

3.1 Effect of different concentration of pectin on the entrapment efficiency of liposomes

The effect of different concentration of pectin on the entrapment efficiency of galanga essential oil liposomes was showed in Fig.1. Similar entrapment efficiency $(32.81 \pm 3.72 \% \sim 35.58 \pm 3.53 \%)$ were obtained after various concentrations of pectin coating. It was probably due to that the pectin forms a thick layer on the surface of liposomes instead of changing the rigidity of the liposomal bilayer. Zhou [3] found that pectin-coated VC nanoliposomes displayed similar entrapment efficiency for different chitosan concentrations, which was similar to our result.

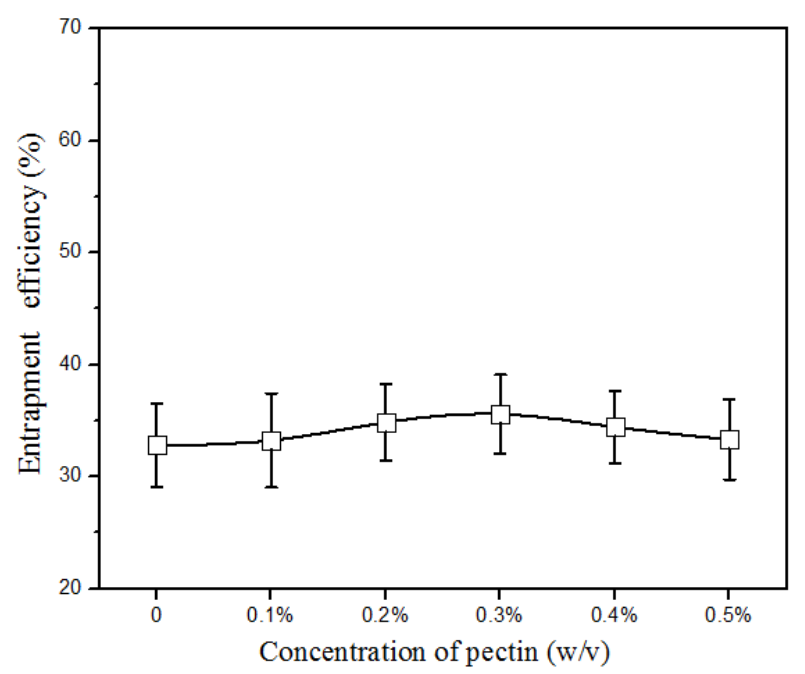

Fig.1 Effect of different concentration of pectin on the entrapment efficiency of galanga essential oil liposomes

3.2 Effect of different concentration of pectin on the average diameter and polydispersity index of liposomes

As is shown in Fig.2, the average diameter and polydispersity index (PDI) of liposomes generally increased with the concentration of pectin increasing. Zhang and Wang [6] suggested polymer could increase the curvature of the liposome particles, indicated by an increase in the thickness of the liposomal layer.

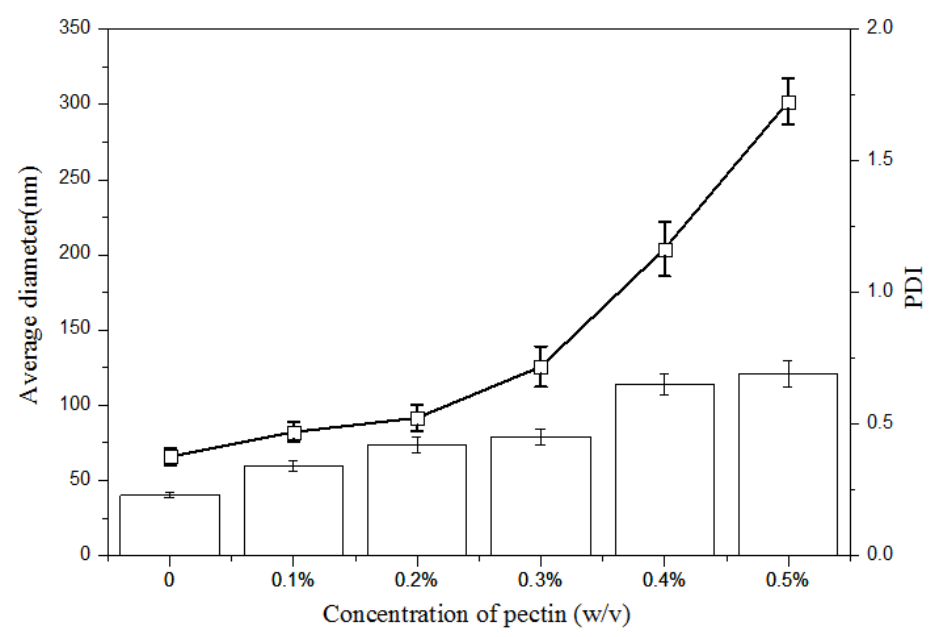

Fig.2 Effect of different concentration of pectin on the average diameter and polydispersity index of galanga essential oil liposomes

3.3 Effect of different concentration of pectin on the average diameter and polydispersity index of liposomes

As is shown in Fig.3, the zeta potential value of liposomes decreased with the concentration of pectin increasing. When the concentration of pectin was more than $0.3 \%(\mathrm{w} / \mathrm{v})$, the zeta potential of liposomes did not change. Since pectin is an anionic polymer, the decrease in zeta potential values of liposomes was probably due to the negatively charged carboxylic group of pectin [7]. When the 
concentration of pectin was more than $0.3 \%(\mathrm{w} / \mathrm{v})$, the zeta potential of liposomes did not change, which was probably due to that the pectin molecules reached saturation on the liposomal surface. Thirawong et al. [8] prepared pectin-liposome nanocomplexes, exhibited similar trend with present study.

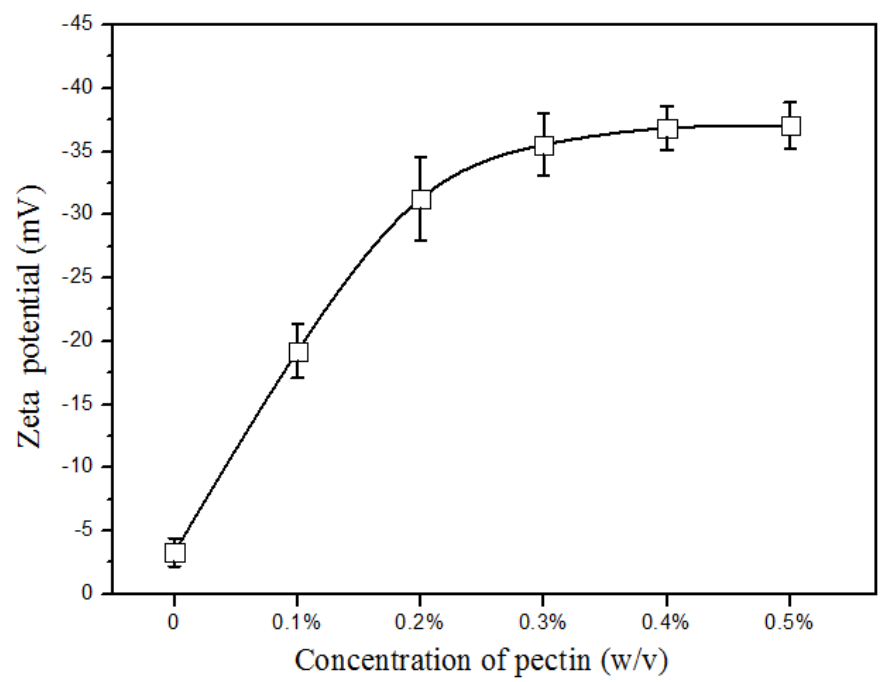

Fig.3 Effect of different concentration of pectin on the zeta potential of galanga essential oil liposomes

\subsection{Storage stability study}

In present study, the physicochemical stability of NL and PNL (pectin concentration $0.3 \%$, w/v) was studied, including zeta potential, average diameter and leakage ratio. The selected samples were storage at $4{ }^{\circ} \mathrm{C}$ and $25^{\circ} \mathrm{C}$ for 10 weeks, and the results were shown in Fig.4-6.

\subsubsection{Change of the zeta potential}

Zeta potential is an important and useful indicator of particle surface charge, which can be used to predict and control the stability of colloidal suspensions or emulsions. From Fig. 4, the zeta potential of all samples changed little at $4{ }^{\circ} \mathrm{C}$ for the whole storage period. When stored at $25{ }^{\circ} \mathrm{C}$, the zeta potential of PNL increased from $-34.72 \mathrm{mV}$ to $-26.85 \mathrm{mV}$, but the zeta potential of NL was still stable. This probably due to the desorption of pectin when stored at $25^{\circ} \mathrm{C}$. Smistad et al [9] studied the change of the zeta potential of pectin-coated liposomes, exhibited similar trend with present study.

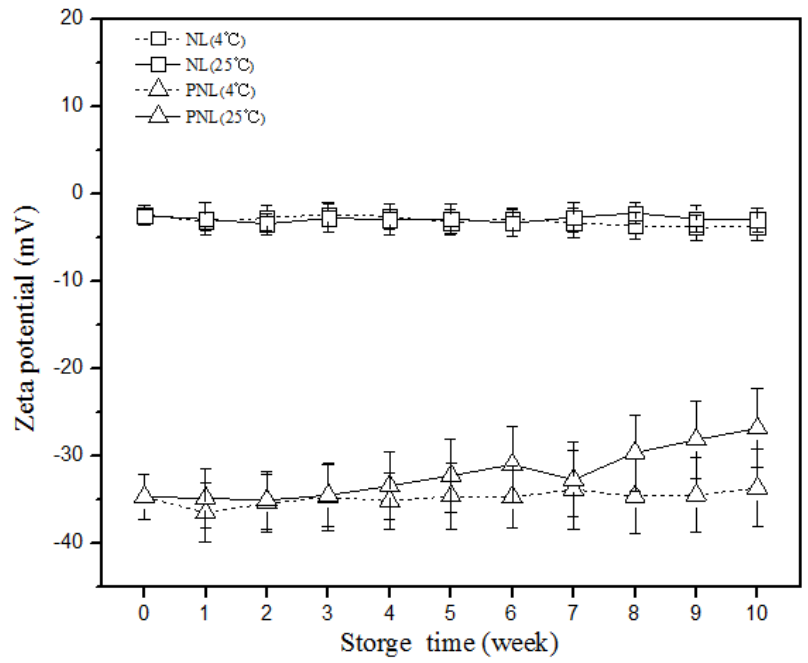

Fig. 4 Change of the zeta potential of liposome samples during storage for 10 weeks

\subsubsection{Change of the average diameter}

As is shown in Fig.5, the average diameter of NL and PNL changed little at $4{ }^{\circ} \mathrm{C}$ for the whole storage period. When stored at $25^{\circ} \mathrm{C}$ after 3 weeks, the average diameter of NL significantly increased, whereas PNL was still stable with these parameters increasing slowly. In detail, the average diameter of NL increased approximately 2.86-fold (from 65.89 to $188.28 \mathrm{~nm}$ ) from beginning to the end of storage at $25{ }^{\circ} \mathrm{C}$. However, the diameter of PNL only increased about 1.34-fold (from 125.62 to 
$168.39 \mathrm{~nm}$ ). The greater absolute value of zeta potential, the higher stable of the suspension, which is because the charged particles repel one another and overcome the natural tendency to aggregate [10]. In this work (Fig. 4), the absolute value of zeta potential of these samples showed $3.31 \mathrm{mV}$ (NL) and $34.72 \mathrm{mV}$ (PNL), respectively, so the PNL presented higher physical stability than NL.

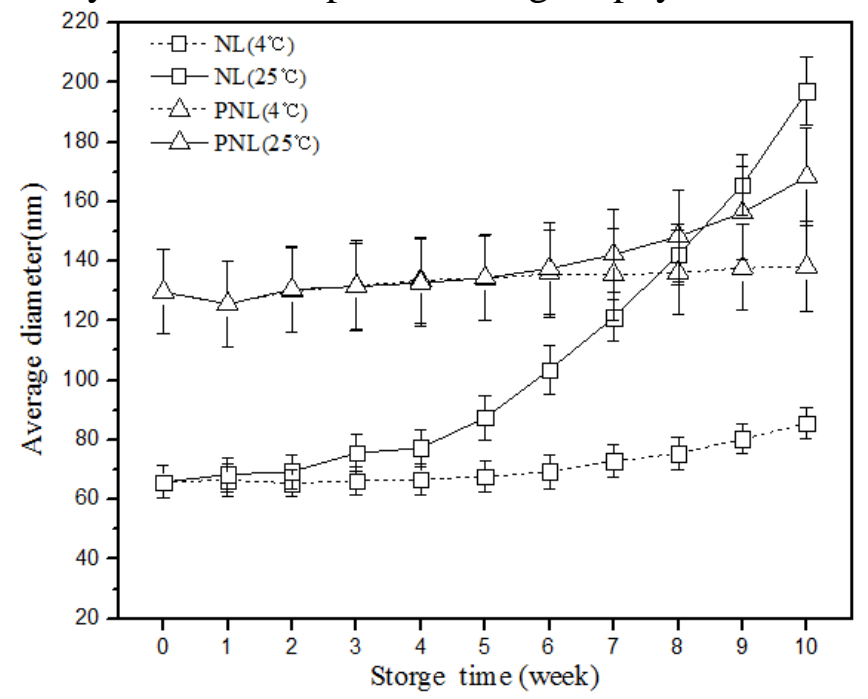

Fig.5 Change of the average diameter of liposome samples during storage for 10 weeks

\subsubsection{Change of the leakage ratio}

As is shown in Fig.5, the leakage of GEO of NL was $60.12 \%$ at the end of storage at $25^{\circ} \mathrm{C}$, in contrast, the PNL was only about $14.68 \%$, respectively. Li et al. [11] stated that the polymers forming a layer around liposome surface could better reduce the oxidation of lipid and prevent the leakage of drugs. Therefore, the PNL showed better chemical stability than NL would be due to the pectin had been successfully coated on the surface of nanoliposomes.

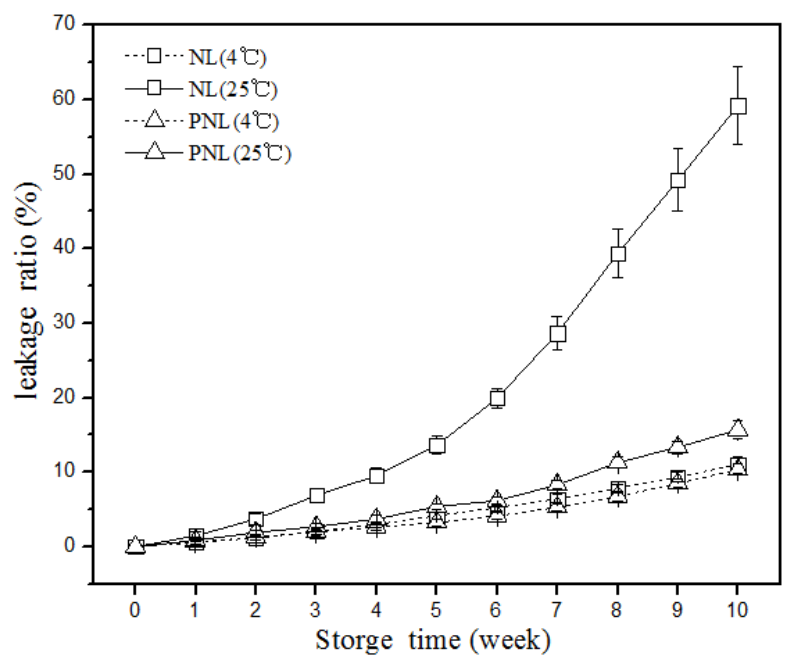

Fig.6 Change of the leakage ratio of liposome samples during storage for 10 weeks

\section{Conclusion}

In this paper, the pectin-coated GEO liposomes can improve storage stability. When the concentration of pectin $(0.3 \%, \mathrm{w} / \mathrm{v}))$, the average diameter of liposomes generally increased from $65.72 \pm 5.81 \mathrm{~nm}$ to $125.62 \pm 13.35 \mathrm{~nm}$, the zeta potential values of liposomes decreased from $-3.31 \pm 1.12 \mathrm{mV}$ to $-35.53 \pm 2.45 \mathrm{mV}$, while the entrapment efficiency of liposomes was similar $(32.81 \pm 3.72 \% \sim 35.58 \pm 3.53 \%)$. Then stored at $4{ }^{\circ} \mathrm{C}$ and $25^{\circ} \mathrm{C}$ for 10 weeks, the pectin-coated GEO liposomes showed better storage stability, with the little change of average diameter and leakage of galanga essential oil. This study can provide technical guidance to the improvement of the stability of galanga essential oil liposomes. 


\section{Acknowledgements}

This research was supported by the National Natural Science Foundation of Hainan province (2015305) and the Science and Technology Plan Projects of Zhanjiang (2014C01005).

\section{References}

[1] Ly T N, Yamauchi R, Shimoyamada M, et al. Isolation and structural elucidation of some glycosides from the rhizomes of smaller galanga (Alpinia officinarum Hance).[J]. Journal of Agricultural \& Food Chemistry, 2002, 50(17):4919-24.

[2] Song B, Liu J X, Liu T. Effect of galangal essential oil on killing human Demodex in vitro.[J]. Journal of Pathogen Biology, 2010.

[3] Zhou W, Liu W, Zou L, et al. Storage stability and skin permeation of vitamin C liposomes improved by pectin coating[J]. Colloids \& Surfaces B Biointerfaces, 2014, 117(5):330-337.

[4] Torchilin V P. Recent advances with liposomes as pharmaceutical carriers[J]. Nature Reviews Drug Discovery, 2005, 4(2):145-60.

[5] Valenti D, De L A, Loy G, et al. Liposome-incorporated santolina insularis essential oil: preparation, characterization and in vitro antiviral activity.[J]. Journal of Liposome Research, 2001, 11(1):73-90.

[6] Zhang J, Wang S L. Topical use of Coenzyme Q10-loaded liposomes coated with trimethyl chitosan: tolerance, precorneal retention and anti-cataract effect.[J]. International Journal of Pharmaceutics, 2009, 372(1-2):66-75.

[7] Park S I, Lee E O, Yang H M, et al. Polymer-hybridized liposomes of poly(amino acid) derivatives as transepidermal carriers.[J]. Colloids \& Surfaces B Biointerfaces, 2013, 110(10):333-338.

[8] Thirawong N, Thongborisute J, Takeuchi H, et al. Improved intestinal absorption of calcitonin by mucoadhesive delivery of novel pectin-liposome nanocomplexes[J]. Journal of Controlled Release, 2008, 125(3):236-45.

[9] Smistad G, Bøyum S, Alund S J, et al. The potential of pectin as a stabilizer for liposomal drug delivery systems[J]. Carbohydrate Polymers, 2012, 90(3):1337-44.

[10] Heurtault B, Saulnier P, Pech B, et al. Physico-chemical stability of colloidal lipid particles[J]. Biomaterials, 2003, 24(23):4283-300.

[11] Li L, Zhang Y, Han S, et al. Penetration enhancement of lidocaine hydrochlorid by a novel chitosan coated elastic liposome for transdermal drug delivery.[J]. Journal of Biomedical Nanotechnology, 2011, 7(7):704-13. 